\title{
Optimization of Maturation of Radio-Cephalic Arteriovenous Fistula Using a Model Relating Energy Loss Rate and Vascular Geometric Parameters
}

\author{
Yang Yang1 (i), Nellie Della Schiava ${ }^{2}$ (D), Pascale Kulisa ${ }^{3}$ (), Mahmoud El Hajem ${ }^{4}$ (), \\ Benyebka Bou-Saïd ${ }^{1}$ (), Serge Simoëns ${ }^{3}$ (), Patrick Lermusiaux ${ }^{2}$ (i)
}

${ }^{1}$ Univ Lyon, CNRS, INSA de Lyon, LaMCoS UMR5259, Villeurbanne, France; ${ }^{2}$ Department of Vascular Surgery, Hospices Civils de Lyon, Lyon, France; ${ }^{3}$ Univ Lyon, CNRS, Ecole Centrale de Lyon, INSA Lyon, Univ Claude Bernard Lyon 1, LMFA, UMR5509, 69130, Ecully, France; ${ }^{4}$ Univ Lyon, INSA Lyon, CNRS, Ecole Centrale de Lyon, Univ Claude Bernard Lyon 1, LMFA, UMR5509, 69621, Villeurbanne, France

Correspondence to: Y. Yang, yang.yang@insa-lyon.fr

Keywords: Hemodialysis, Radio-Cephalic Arteriovenous Fistula (RCAVF), Critical Energy Loss Rate (CEL), Vessel Geometric Parameters, Maturity

Received: April 28, $2021 \quad$ Accepted: June 22, $2021 \quad$ Published: June 25, 2021

Copyright $\odot 2021$ by author(s) and Scientific Research Publishing Inc.

This work is licensed under the Creative Commons Attribution International License (CC BY 4.0).

http://creativecommons.org/licenses/by/4.0/

\section{(c) (i) Open Access}

\section{ABSTRACT}

The main reason for the early failure of radio-cephalic arteriovenous fistula (RCAVF) is non-maturity, which means that the blood flow rate in the fistula cannot increase to the expected value for dialysis. From a mechanical perspective, the vascular resistance at the artificially designed anastomosis causes an energy loss that affects blood flow rate growth and leads to early failure. This research studied how to maximize the RCAVF maturity and primary patency by controlling the energy loss rate. We theoretically analyzed and derived a model that evaluates the energy loss rate $E_{\text {avf }}$ in RCAVF as a function of its blood vessel geometric parameters (GPs) for given flow rates. There was an aggregate of five controllable GPs in RCAVF: radial artery diameter $\left(D_{r a}\right)$, cephalic vein diameter $\left(D_{c v}\right)$, blood vessel distance between artery and vein $(h)$, anastomotic diameter $\left(D_{a}\right)$, and anastomotic angle $(\theta)$. Through this analysis, it was found that $E_{a v f}$ was inversely proportional to $D_{r a}, D_{c r} D_{a}$, and $\theta$, whereas proportional to $h$. Therefore, we recommended surgeons choose the vessels with large diameters, close distance, and increase the diameter and angle of the anastomosis to decrease the early failure of RCAVF. Simultaneously, we could explain the results of many clinical empiricisms with our formula. We found that increasing $D_{c v}$ and $\theta$ was more significant in reducing $E_{\text {avf }}$ than increasing $D_{r a}$ and $D_{a}$. Based on our model, we could define two critical energy loss rates $\left(C E L_{a}, C E L_{b}\right)$ to help surgeons evaluate the blood vessels and 


\section{choose the ideal range of $\theta$, and help them design the preoperative RCAVF plan for each patient to increase the maturity and the primary patency of RCAVF.}

\section{INTRODUCTION}

Patients with end-stage renal disease (ESRD) need to undergo hemodialysis (HD) to survive. The arteriovenous fistula is the gold standard for vascular access for hemodialysis, and it is the access recommended by the NFKDOQI [1]. Among all possible AVFs, distal RCAVF is recommended as the first choice of native AVFs. However, due to its small flow capability and thin vessels, the venous outflow after the operation cannot reach the expected blood flow. It will lead to the non-maturation of RCAVF and causes a higher early failure rate. Therefore, the choice of radial artery (RA) and cephalic vein (CV) and the design of the anastomosis play an essential role in the postoperative maturity rate of RCAVF.

We proposed in this study a model to optimize the design of the RCAVF. This new complete model using the interrelation between the different GPs brought scientific probes about the hemodynamic of RCAVF. Our research was inspired by Murray's law [2]. The geometric structure of human blood vessels has evolved according to natural biological selection. The structure of blood vessels follows the principle of minimum energy loss. AVF is an artificial connection of blood vessels. When the energy loss generated by the designed vascular circuit is small, the blood flow is easier to establish so that venous blood flow can reach the target value more easily and quickly, which facilitates the maturation of the AVF.

The research review [3] summarized previous studies and recommended surgeons to choose $D_{r a}$ and $D_{c V}$ equal or greater than $2 \mathrm{~mm}$, and avoid vessels less than or equal to $1.5 \mathrm{~mm}$ to ensure the maturity of RCAVF. References [4-6] pointed out that the RCAVF maturity is closely related to $D_{r a}$ and $D_{c v}$. References [7-11] are based on numerical simulations of different RCAVF shapes to enforce that $\theta$ has an impact on the hemodynamic distribution in a fistula by observing the wall shear stress. However, these previous works do not offer theoretical explanations, and literature about the hemodynamic conditions in a recent AVF remains poor. Analyses are often carried out to impact variables separately without considering at least their cross-correlated influences or the interaction with other geometric factors such as $h$ and $D_{a}$. Actually, surgeons apply some guidelines and have some surgical habits, but with very few scientific probes, and the AVF creation varies from center to center. Our research has considered a set of five a priori inter-connected geometric variables in RCAVF through the control of different GPs to improve recommendations for the creation of RCAVF.

\section{METHODS}

\subsection{Theoretical Analysis}

Before the RCAVF creation, surgeons measured and analyzed the quality of the patient vessels through duplex ultrasound and selected the a priori most appropriate AVF according to the $D_{r a}$ and $D_{c r}$ based on their clinical experiences. During the operation, surgeons release the vein over a certain distance to bring the vein to the artery, then cut the vein (CV) obliquely following an angle $\theta$ and cut along the axial direction of the artery (RA) a length $D_{a}$ as shown in Figure 1(a). Then RA and CV will be connected to form the RCAVF vascular access, as depicted in Figure 1(b). The blood flow in the proximal artery (PA) enters the fistula through the heel of the anastomosis with a flow rate $Q_{h}$ equivalent to that of the normal radial artery $(22 \mathrm{~mL} / \mathrm{min})$. The blood flow in the distal artery (DA) enters the fistula through the toe of the anastomosis at a flow rate $Q_{t}$ with the same order of magnitude as that of the wrist $(5 \mathrm{~mL} / \mathrm{min}) . Q_{h}$ and $Q_{t}$ were calculated by multiplying the average flow velocity in the blood vessel by the cross-section of the vessel [12-14].

$E_{a v f}$ in the RCAVF included energy lost due to vessel bending $\left(E_{b}\right)$ and friction $\left(E_{f}\right)$ in $\mathrm{CV}$, as well as the dissipation of energy due to change in the direction of blood flow $\left(E_{d}\right)$ at the anastomosis. In this research, the blood was laminar flow, and the following assumptions were used: 


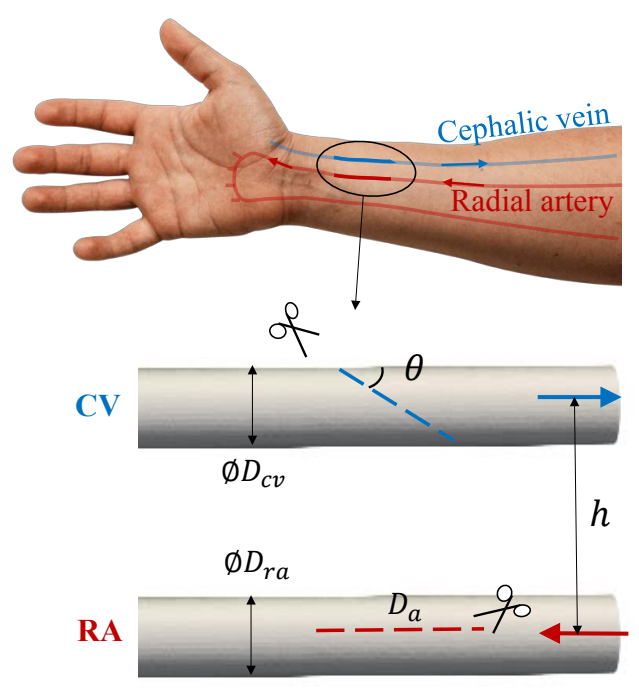

(a)

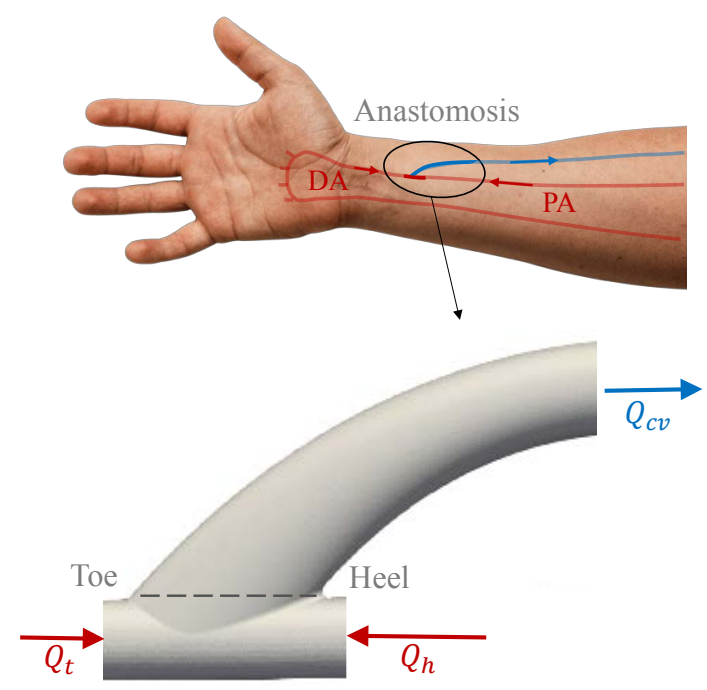

(b)

Figure 1. Radio-cephalic arteriovenous fistula (RCAVF) schematic diagram and geometric parameters (GPs).

- Blood is an incompressible Newtonian fluid $\left(\rho=1060 \mathrm{~kg} / \mathrm{m}^{3}, \mu=0.0035 \mathrm{~Pa} \cdot \mathrm{s}\right)$ flowing in a rigid horizontal blood vessel;

- The potential energy and the heat transfer are negligible;

- The energy loss due to metabolism is negligible.

\subsubsection{Energy Loss Rate in Bending Cephalic Vein}

The energy loss rate along a bending CV segment (Figure 2) consists of $E_{b}$ and $E_{f}$. We assumed that this segment is a concentric bending vessel with a constant diameter $D_{c r}$ In [15], Idelchik defined $E_{b}$ as:

$$
E_{b}=\zeta_{M} \cdot \frac{\rho Q_{c v}^{3}}{2 S_{c v}^{2}},
$$

where $\zeta_{M}$ is the minor loss coefficient of the bending vessel, it can be defined as:

$$
\zeta_{M}=A_{1} B_{1} C_{1} \text {. }
$$

$A_{1}, B_{1}$, and $C_{1}$ are defined by:

$$
\left\{\begin{array}{l}
A_{1}=0.9 \sin \theta \text { when } \theta \leq 90^{\circ} \\
B_{1}=0.21\left[\frac{D_{c v}}{R_{0}}\right]^{i}, R_{0}=\frac{h-D_{r a} / 2}{1-\cos \theta} \\
C_{1}=1
\end{array}\right.
$$

$R_{0}$ is the radius of curvature of the bending segment. When the ratio of $D_{c v}$ to $R_{0}$ is less than $1, i$ is equal to 2.5; otherwise, $i$ is equal to 0.5. Finally, from Equations (1) to (3), the energy loss rate of the CV segment due to bending could be summarized as:

$$
E_{b}=0.189 \sin \theta\left[\frac{D_{c v}(1-\cos \theta)}{h-D_{r a} / 2}\right]^{i} \frac{\rho Q_{c v}^{3}}{2 S_{c v}^{2}} .
$$

When blood transports in vessels, the frictional forces will cause pressure loss, thereby consuming energy. The energy loss rate due to friction [2] in this segment could be expressed as: 


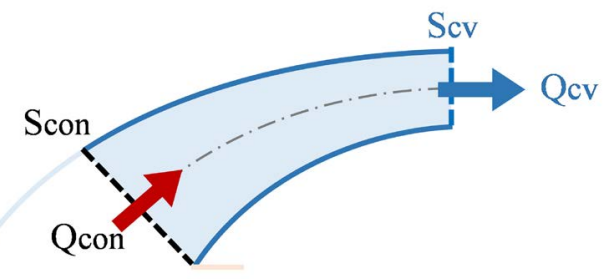

Figure 2. Blood flow in bending cephalic vein.

$$
E_{f}=\frac{128 \mu\left(h-D_{r a} / 2\right) \theta Q_{c v}^{2}}{\pi D_{c v}^{4}(1-\cos \theta)} .
$$

\subsubsection{Energy Loss rate at Anastomosis}

Placement of the fistula allows the blood flow in RA to bypass the distal resistance generated by the capillary in the fingers and return to the heart through the anastomosis. For that reason, $Q_{t}$ flow rate direction of the distal artery changes and flows into the vein together with $Q_{h}$ (Figure 3). $Q_{c o n}$ represents the total blood flow rate at the confluence, which equals $Q_{c r}$. The resulting energy loss rate at anastomosis could be expressed as:

$$
E_{a}=E_{t}+E_{h}=\left(K_{t}+K_{h}\right) \frac{\rho U_{c o n}^{2}}{2} Q_{c o n}=\left(K_{t}+K_{h}\right) \frac{\rho Q_{c v}^{3}}{2 S_{c o n}^{2}} .
$$

The minor loss coefficients at toe and heel are $K_{t}$ and $K_{h}[16]$ defined by:

$$
\begin{aligned}
K_{t} & =2\left(1-q_{t}\right)^{2} \frac{1}{\varphi_{t}} \cos \left(\frac{\pi+3 \theta}{4}\right)-2 q_{t}^{2} \frac{1}{\varphi_{t}} \cos \left(\frac{3 \theta}{4}\right)+q_{t}^{2} \frac{1}{\varphi_{t}}+1 \\
K_{h} & =2 q_{h}^{2} \frac{1}{\varphi_{h}} \cos \left(\frac{\pi+3 \theta}{4}\right)-2\left(1-q_{h}\right)^{2} \frac{1}{\varphi_{h}} \cos \left(\frac{3 \theta}{4}\right)+q_{h}^{2} \frac{1}{\varphi_{h}}+1,
\end{aligned}
$$

where $q_{t}$ and $q_{h}$ are the ratios of the toe and the heel volume flow rate to the common flow rate. $\varphi_{t}$ and $\varphi_{h}$ are the ratios of the toe and the heel cross-sectional areas to the common one. They can be expressed as:

$$
\left\{\begin{array}{l}
q_{t}=\frac{Q_{t}}{Q_{c o n}} \\
q_{h}=\frac{Q_{h}}{Q_{c o n}}
\end{array},\left\{\begin{array}{l}
\varphi_{t}=\frac{S_{t}}{S_{c o n}} \\
\varphi_{h}=\frac{S_{h}}{S_{c o n}}
\end{array} .\right.\right.
$$

Next, we introduced the calculation of the cross-sectional area of the total blood flow at the confluence $\left(S_{c o n}\right)$, as shown in Figure 4 . In the anastomosis operation, surgeons cut CV obliquely to obtain an elliptical cross-section $S_{a_{-} c V}$ (the light blue ellipse in Figure 4), the major axis is $A_{a_{-} c r}$ and the minor axis is $B_{a_{-} c r}$ :

$$
A_{a_{-} c v}=\frac{D_{c v}}{\sin \theta}, \quad B_{a_{-} c v}=D_{c v} .
$$

Then RA is cut along the axial direction and opened along the radial direction of the blood vessel to obtain an elliptical anastomosis $S_{a}$ with a major axis $A_{a}$ and a minor axis $B_{a}\left(A_{a}\right.$ is equal to the anastomotic diameter $D_{a}$, and $B_{a}$ is equal to RA radius $R_{r a}$ ). In order to suture CV and RA, $S_{a-c v}$ needs to be stretched or compressed along the major axis to give it the same shape as $S_{a}$ (the red ellipse in Figure 4). The $S_{\text {con }}$ section is perpendicular to the $\mathrm{CV}$ axial direction after the $\mathrm{CV}$ is stretched or compressed (the black ellipse in 


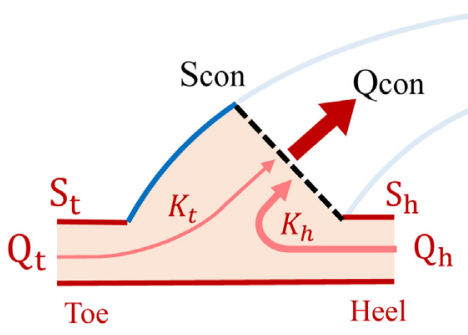

Figure 3. Blood flow at anastomosis.

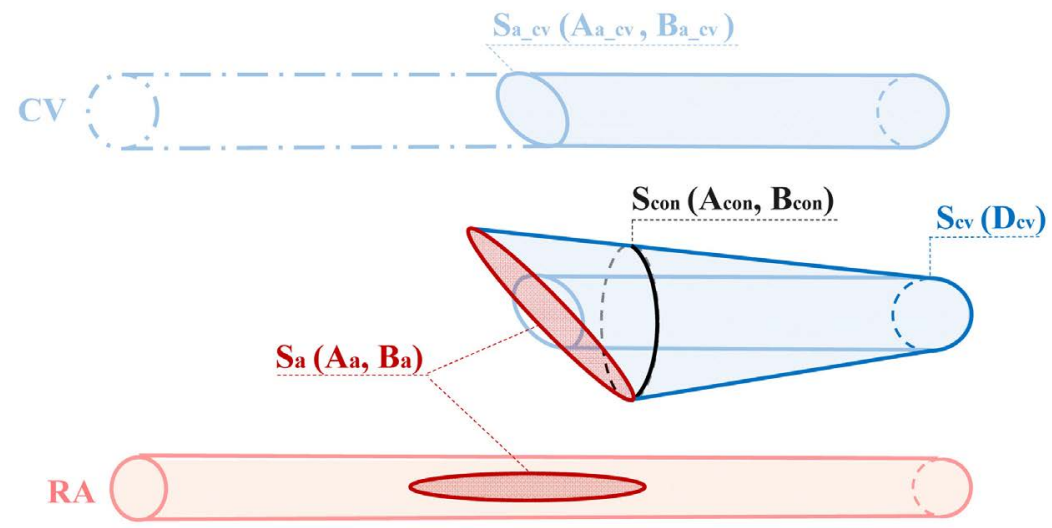

Figure 4. 3D simplified diagram of the anastomosis operation. Top is the original CV artery before being stretched (middle) to be linked to the RA (bottom).

Figure 4 with the major axis $A_{c o n}$ and the minor axis $\left.B_{c o n}\right) . S_{c v}$ in Figure 4 represents the cross-section of $\mathrm{CV}$, a circular cross-section with a diameter of $D_{c r}$. Figure 5 presents the top view and the front view of the blood vessel in the CV stretch segment (the light blue lines in Figure 5(a) and Figure 5(c) represent an undeformed vein). We supposed that $B_{a}$ and $B_{c o n}$ evolved linearly from $D_{c n}$ and $A_{c o n}$ could be calculated from $A_{a}, D_{c n}$ and the $\theta$ angle of the imposed deformation. After geometric calculations, the expression of $A_{c o n}$ and $B_{c o n}$ were:

$$
\left\{\begin{array}{l}
A_{c o n}=D_{a} \sin \theta-\frac{\left(D_{a} \sin \theta-D_{c v}\right) D_{a} \cos \theta}{2 h+D_{a} \sin \theta(1-\cos \theta)} \\
B_{c o n}=\frac{D_{r a}}{2}+\frac{\left(2 D_{c v}-D_{r a}\right) D_{a} \cos \theta(1-\cos \theta)}{4 h \theta}
\end{array}\right.
$$

Therefore, the cross-section of the total blood flow at the $S_{c o n}$ confluence could be obtained from:

$$
S_{c o n}=\frac{\pi A_{c o n} B_{c o n}}{4} \text {. }
$$

\subsubsection{Total Energy Loss Rate in RCAVF}

From Equations (4), (5), and (5), the total energy loss rate in RCAVF ( $\left.E_{\text {avf }}\right)$ was given by the following equation:

$$
\begin{aligned}
E_{\text {avf }} & =E_{b}+E_{f}+E_{a} \\
& =0.189 \sin \theta \sqrt{\frac{D_{c v}(1-\cos \theta)}{h-D_{r a} / 2}} \frac{\rho Q_{c v}^{3}}{2 S_{c v}^{2}}+\frac{128 \mu\left(h-D_{r a} / 2\right) \theta Q_{c v}^{2}}{\pi D_{c v}^{4}(1-\cos \theta)}+\left(K_{t}+K_{h}\right) \frac{\rho Q_{c v}^{3}}{2 S_{c o n}^{2}}
\end{aligned}
$$

where $K_{t}, K_{h}$, and $S_{c o n}$ could be obtained by Equations (7), (8), and (12). 


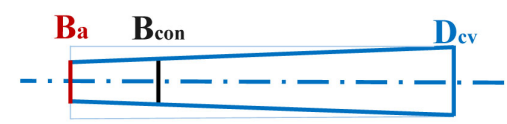

(a)

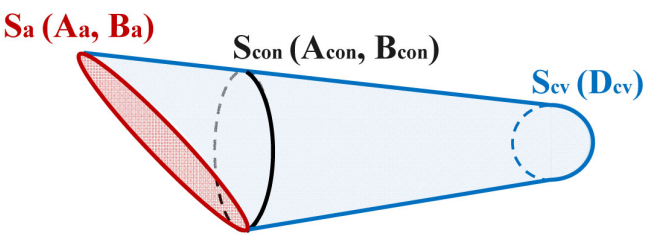

(b)

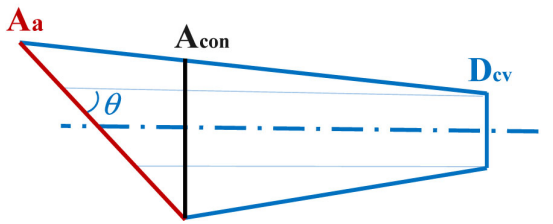

(c)

Figure 5. Top view, 3D view, and front view of cephalic vein (CV) after stretching. (a) Top view; (b) $3 \mathrm{D}$ view; (c) Front view.

\subsection{Model Validation}

In order to validate the model, we compared $E_{\text {avf }}$ calculated by Equation (13) and $E_{\text {avfnum }}$ obtained with Foam-extend 4.0 numerical simulations. To ensure the relevance of the model, the comparison was made for 25 RCAVF 3D shapes created with Catia V5.

In Equation (13), there was a total of 5 GPs $\left(D_{r a}, D_{c b} h, D_{a}\right.$, and $\left.\theta\right)$. We created a standard model (as a reference of fixed values of GPs as shown in Table 1), then only changed one GP each time. For a given GP, we selected five values within its range (Table 1) and kept the remaining GPs consistent with the standard model. Therefore, we created a total of 25 models. The details of the models are shown in Table 2.

In foam-extend 4.0, we used pisoFoam [18] solver to simulate the flow in these geometries with the same initial conditions (flow rate and pressure). After numerical convergence, the pressure at anastomosis inlets $\left(S_{t}, S_{h}\right)$ and outlet $\left(S_{c v}\right)$ (Figure 6) could be extracted. They denoted respectively $P_{b}, P_{h}$, and $P_{c r}$. According to the law of energy conservation, $E_{\text {avfnum }}$ of each model can be calculated by:

$$
\frac{\rho}{2} \frac{Q_{t}^{3}}{S_{t}^{2}}+P_{t} Q_{t}+\frac{\rho}{2} \frac{Q_{h}^{3}}{S_{h}^{2}}+P_{h} Q_{h}=\frac{\rho}{2} \frac{Q_{c v}^{3}}{S_{c v}^{2}}+P_{c v} Q_{c v}+E_{\text {avf } \_ \text {num }} .
$$

$Q_{t}$ and $Q_{h}$ are the flow rates at arterial inlets (Table 2). According to mass conservation, $Q_{c v}$ is equal to the sum of $Q_{t}$ and $Q_{h}$.

\section{RESULTS AND DISCUSSIONS}

\subsection{Comparison between Numerical and Analytical Results}

We calculated $E_{\text {avf }}$ for 25 models using Equation (13) and compared them with $E_{\text {avf_num }}$ obtained by numerical simulations. The comparisons were shown in Figure 7. The blue dots in Figure 7 represent the analytical results $\left(E_{\text {avf }}\right)$, and the red dots represent the numerical results $\left(E_{\text {avfnum }}\right)$. We could observe that, for each geometric parameter, $E_{\text {avfnum }}$ and $E_{\text {avf }}$ had the same profile. The relative difference between the two results defined as $\left(\left|E_{\text {avf }}-E_{\text {avf_num }}\right| / E_{\text {avf }}\right)$ was shown in Table 3 for each case. The relative difference between the two approaches was less important when analyzing the impact of the variation of $D_{c v}$ or h. It varied between $1 \%$ and $7 \%$ for $D_{c r}$. For h, it oscillated between $1 \%$ and $9 \%$. In the case of $D_{r a}$ and $D_{a}$, the relative difference was greater and reached $12 \%$. In all cases, the difference between the two approaches was 


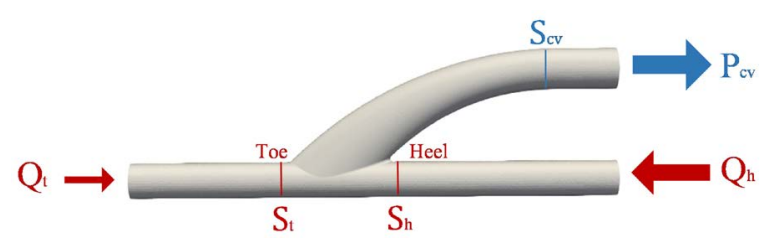

Figure 6. RCAVF simulation model.

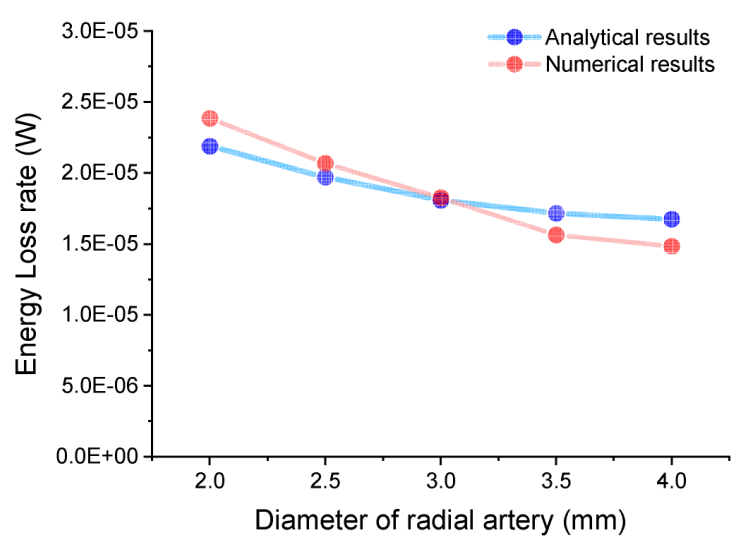

(a)

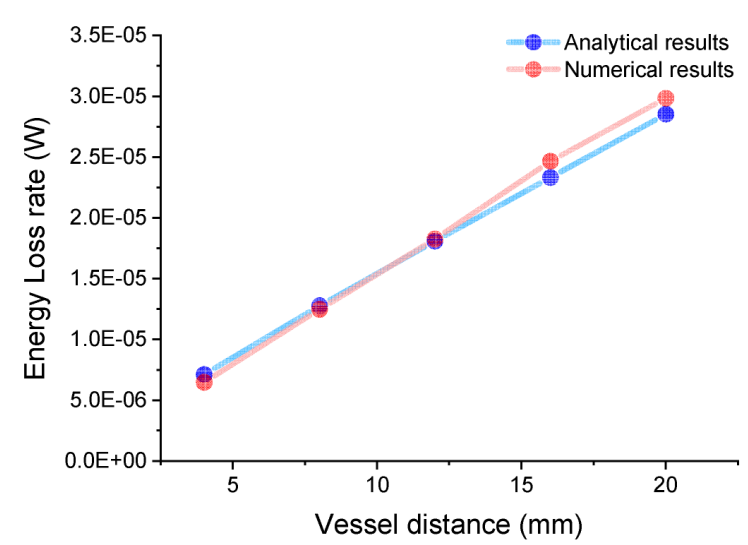

(c)

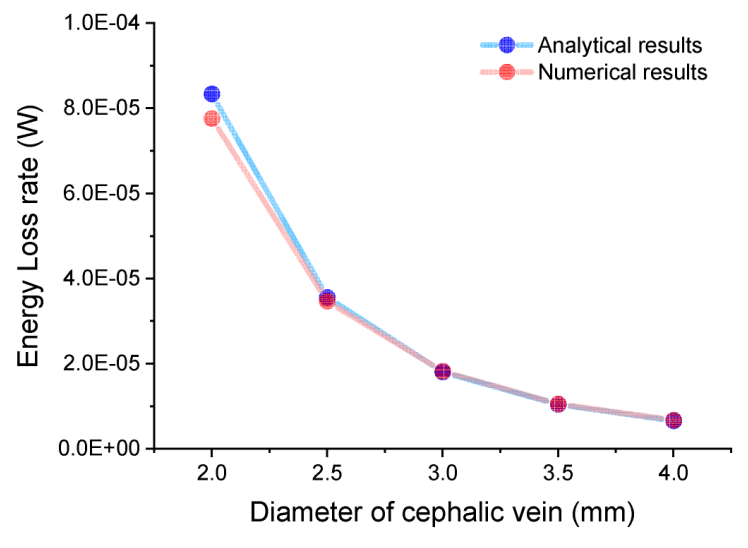

(b)

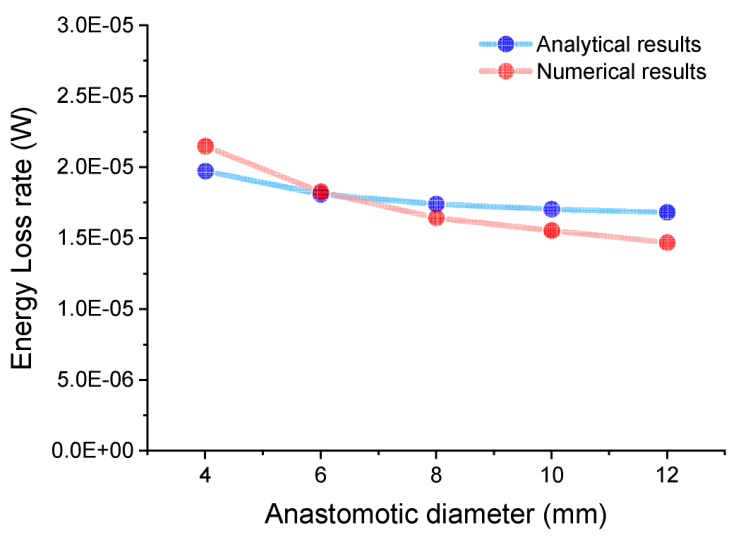

(d)

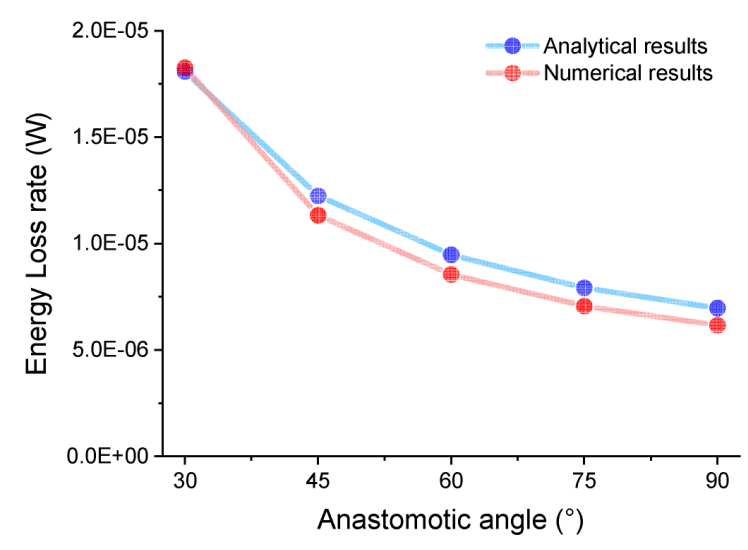

(e)

Figure 7. Comparison of numerical results and analytical results in 25 models. 
Table 1. The research ranges of 5 GPs.

\begin{tabular}{cccccc}
\hline GPs & $D_{r a}(\mathrm{~mm})$ & $D_{c V}(\mathrm{~mm})$ & $h(\mathrm{~mm})$ & $D_{a}(\mathrm{~mm})$ & $\theta\left(^{\circ}\right)$ \\
\hline Research range & {$[1,4]$} & {$[1,4]$} & {$[5,50]$} & {$[4,12]$} & {$[10,90]$} \\
\hline
\end{tabular}

Table 2. Parameters of simulation models.

\begin{tabular}{|c|c|c|c|c|c|c|}
\hline \multirow{2}{*}{ Models } & \multicolumn{5}{|c|}{ GPs } & \multirow{2}{*}{ Initial Condition } \\
\hline & $D_{r a}(\mathrm{~mm})$ & $D_{c V}(\mathrm{~mm})$ & $h(\mathrm{~mm})$ & $D_{a}(\mathrm{~mm})$ & $\theta\left(^{\circ}\right)$ & \\
\hline Standard Model & 3 & 3 & 12 & 6 & 30 & \\
\hline \multirow{5}{*}{$\begin{array}{l}\text { Model } 1 \text { - } 5 \\
\text { ( } D_{r a} \text { change) }\end{array}$} & 2 & & & & & \\
\hline & 2.5 & & & & & \\
\hline & 3 & 3 & 12 & 6 & 30 & \\
\hline & 3.5 & & & & & \\
\hline & 4 & & & & & \\
\hline \multirow{5}{*}{$\begin{array}{l}\text { Model } 6 \text { - } 10 \\
\left(D_{c V} \text { change }\right)\end{array}$} & & 2 & & & & \\
\hline & & 2.5 & & & & \\
\hline & 3 & 3 & 12 & 6 & 30 & \\
\hline & & 3.5 & & & & \\
\hline & & 4 & & & & \\
\hline \multirow{5}{*}{$\begin{array}{l}\text { Model } 11-15 \\
\text { ( } h \text { change })\end{array}$} & & & 4 & & & $Q_{t}=5 \mathrm{~mL} / \mathrm{min} ;$ \\
\hline & & & 8 & & & $Q_{h}=22 \mathrm{~mL} / \mathrm{min}$ \\
\hline & 3 & 3 & 12 & 6 & 30 & $P_{c V}=8.7 \mathrm{mmHg}$ \\
\hline & & & 16 & & & (CV outlet pressure [17]) \\
\hline & & & 20 & & & \\
\hline \multirow{5}{*}{$\begin{array}{l}\text { Model } 16-20 \\
\left(D_{a} \text { change }\right)\end{array}$} & & & & 4 & & \\
\hline & & & & 6 & & \\
\hline & 3 & 3 & 12 & 8 & 30 & \\
\hline & & & & 10 & & \\
\hline & & & & 12 & & \\
\hline \multirow{5}{*}{$\begin{array}{l}\text { Model } 21-25 \\
\quad(\theta \text { change })\end{array}$} & & & & & 30 & \\
\hline & & & & & 45 & \\
\hline & 3 & 3 & 12 & 6 & 60 & \\
\hline & & & & & 75 & \\
\hline & & & & & 90 & \\
\hline
\end{tabular}


Table 3. Relative difference between numerical results and analytical results.

\begin{tabular}{ccccccccccc}
\hline Models & 1 & 2 & 3 & 4 & 5 & 6 & 7 & 8 & 9 & 10 \\
\hline Relative Difference (\%) & 8.99 & 4.97 & 0.94 & 8.86 & 11.38 & 6.92 & 2.41 & 0.94 & 1.06 & 2.50 \\
Model & 11 & 12 & 13 & 14 & 15 & 16 & 17 & 18 & 19 & 20 \\
Relative Difference (\%) & 9.03 & 2.29 & 0.94 & 5.71 & 4.58 & 8.84 & 0.94 & 5.59 & 8.81 & 12.64 \\
Models & 21 & 22 & 23 & 24 & 25 & & & & & \\
Relative Difference (\%) & 0.94 & 7.49 & 9.80 & 10.82 & 11.61 & & & & &
\end{tabular}

minimal for the standard value of the geometric variables. The difference became greater when the geometric variables moved away from the reference values. Whereas for the $\theta$, the difference between the numerical approach and the model tended to increase from $1 \%$ to $11 \%$ when the angle increased.

These differences could be explained by the simplifications of the geometry of the RCAVF made for this study. Another possible explanation was linked to the assumptions made initially in [16] on which we relied to establish the expression of $E_{a}$ given by Equation (6). Nevertheless, we could consider that these differences between $E_{\text {avf }}$ and $E_{\text {num_avf }}$ remain within an acceptable range. Therefore, Equation (13) established here could reasonably be exploited to predict the energy loss rate in RCAVF.

\subsection{Relationship between $E_{A V F}$ and GPs}

We used Matlab2017 to analyze deeper the relationship between $E_{\text {avf }}$ and the different GPs. The curves illustrating this relationship are represented in Figures 8-12. For each figure, the evolution of $E_{a v f}$ as a function of a GP was illustrated. For each GP, the evolution of the $E_{\text {avf }}$ was parametrized by one of the remaining GPs (subfigures a to d). The three remaining GPs were kept constant and equaled to their respective value corresponding to the standard model given in Table 2.

Thus, Figure 8 shows the relationship between $E_{a v f}$ and $D_{r a}$. In Figure 8(a), we set $h, D_{a}$ and $\theta$ to the standard constant values and changed $D_{c r}$. From this figure, we observed that for all the values of $D_{c n}$ the $E_{\text {avf }}$ decreased when the $D_{r a}$ increased. For a fixed value of $D_{r a}$, the energy loss rate was lower when the $D_{c r}$ increased. The decrease in $E_{a v f}$ as a function of $D_{r a}$ was more pronounced for the smaller $D_{c v}$ values. This general tendency to have lower losses as the diameter of the artery or vein increases could be explained by lower blood velocities at constant flow and larger diameters. As a result, the friction decreased.

In Figure 8(b), we set $D_{c r} . D_{a}$, and $\theta$ to the constant values and changed h. The behavior of $E_{a v f}$ versus $D_{r a}$ was the same as in the previous case. The energy loss rate decreased when $D_{r a}$ increased. Here, on the other hand, for a fixed value of $D_{r a}$, the energy loss was more significant when the distance $h$ increased. This result seemed consistent since the friction loss rate was proportional to the distance traveled by the blood. A greater distance $\mathrm{h}$ implied a greater length of the bending vein, so a more significant energy loss rate appeared.

Figure 8(c) portrayed the relationship curve between $E_{a v f}$ and $D_{r a}$ when $D_{c r} h$, and $\theta$ were unchanged, and $D_{a}$ was changed. Here the behavior was the same as for Figure 8(a). A larger diameter was the source of a more limited energy loss rate.

Figure 8(d) gives the evolution of $E_{a v f}$ as a function of $D_{r a}$ and $\theta$ when $D_{c r}, h$, and $D_{a}$ are unchanged. The decrease in the angle accentuated the decrease in $E_{\text {avf }}$ as a function of the diameter.

Without detailing the analysis of all Figures 8-12, it was possible to conclude that we had the following general tendencies:

1) $E_{\text {avf }}$ was inversely proportional to $D_{r a}, D_{c r}, D_{a}$, and $\theta$, and proportional to $h$. In other words, if surgeons want to improve the maturity rate of RCAVF, we suggested them choose RA and CV with larger diameters and closer distance and make an anastomotic plan with a larger anastomotic diameter and anastomosis angle. 


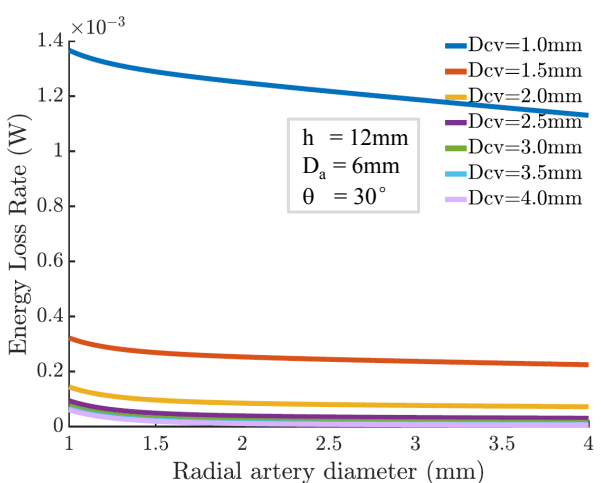

(a)

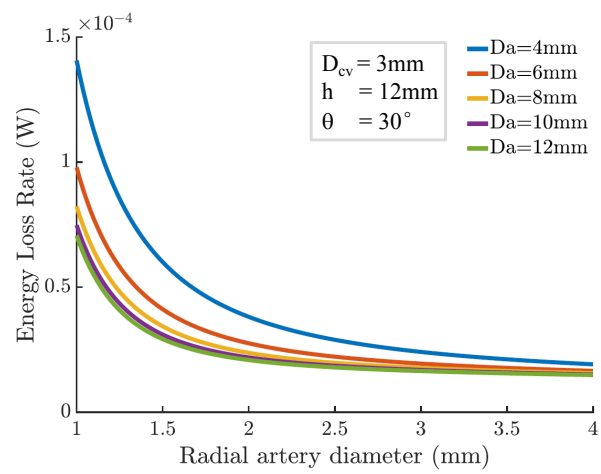

(c)

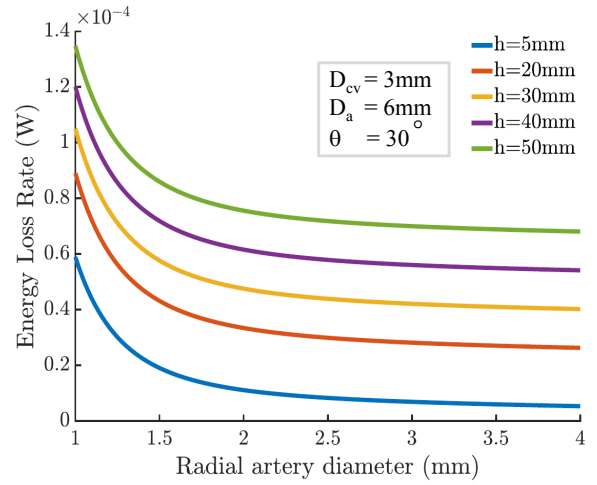

(b)

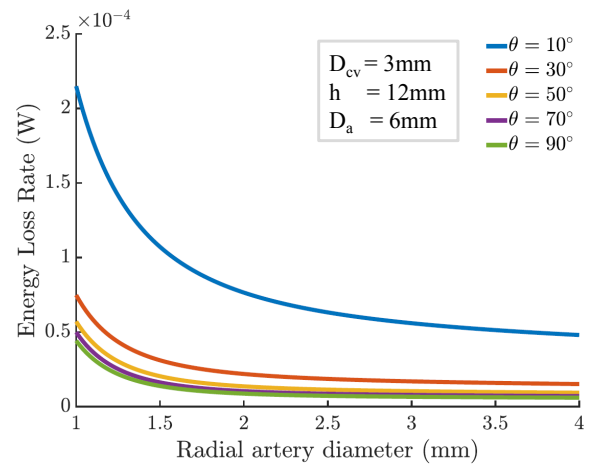

(d)

Figure 8. The relationship between energy loss $\left(E_{\text {avf }}\right)$ and radial artery diameter $\left(D_{r a}\right)$.

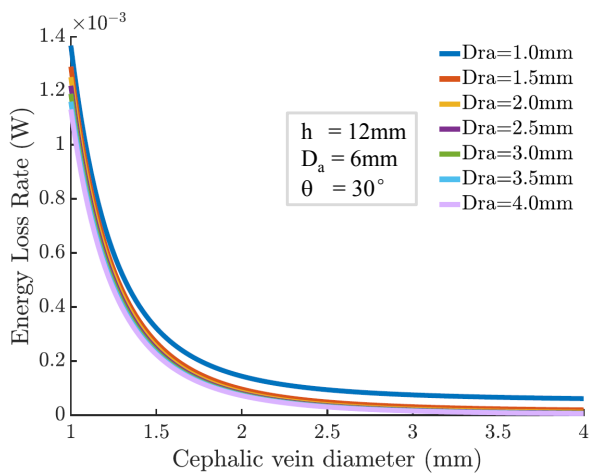

(a)

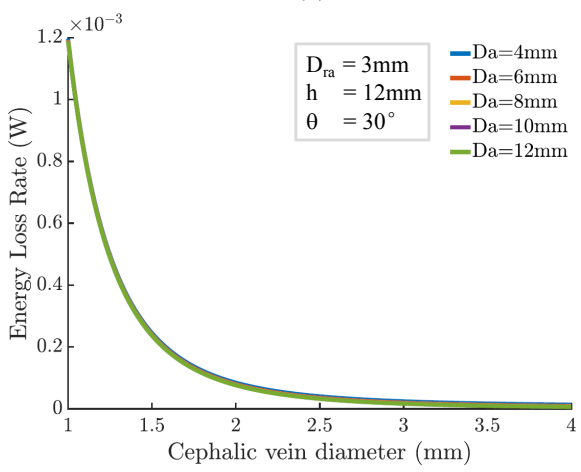

(c)

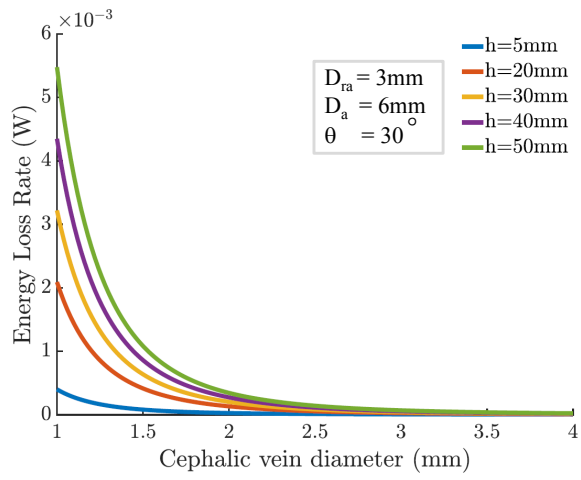

(b)

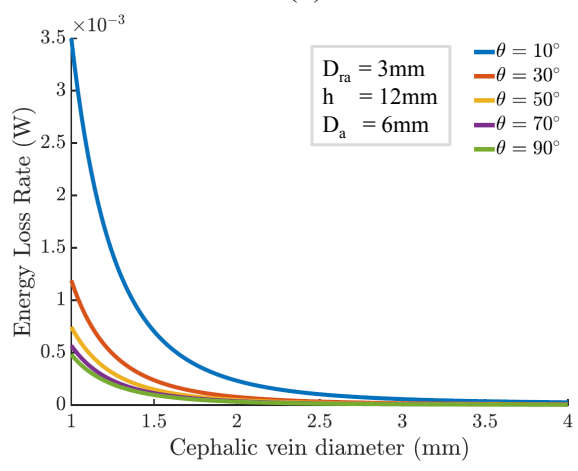

(d)

Figure 9. The relationship between energy loss $\left(E_{\text {avf }}\right)$ and cephalic vein diameter $\left(D_{c v}\right)$. 


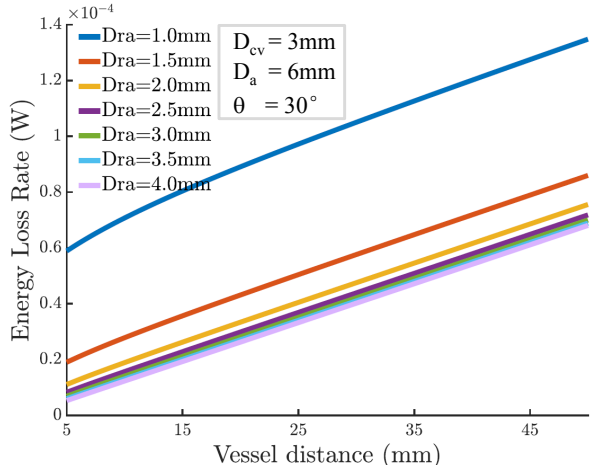

(a)

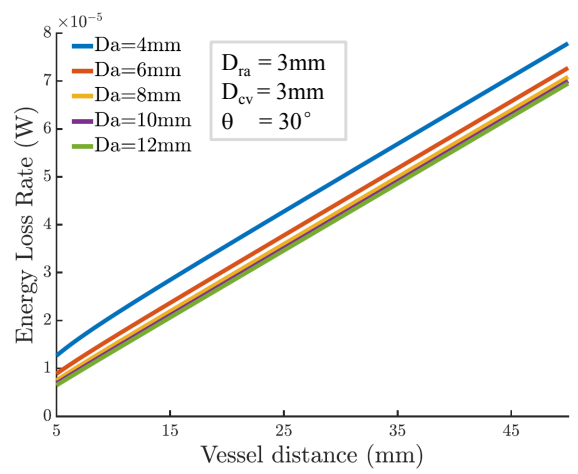

(c)

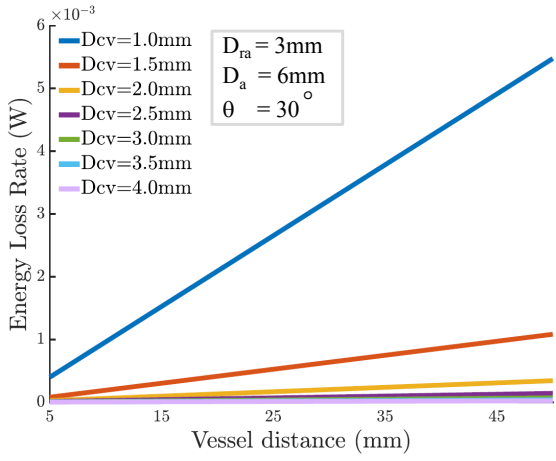

(b)

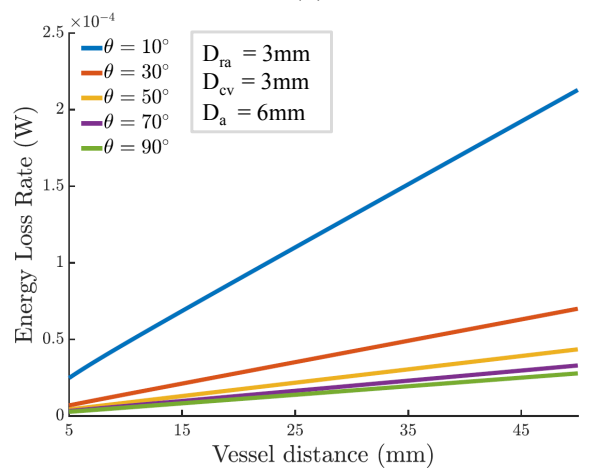

(d)

Figure 10. The relationship between energy loss $\left(E_{\text {avf }}\right)$ and vessel distance $(h)$.

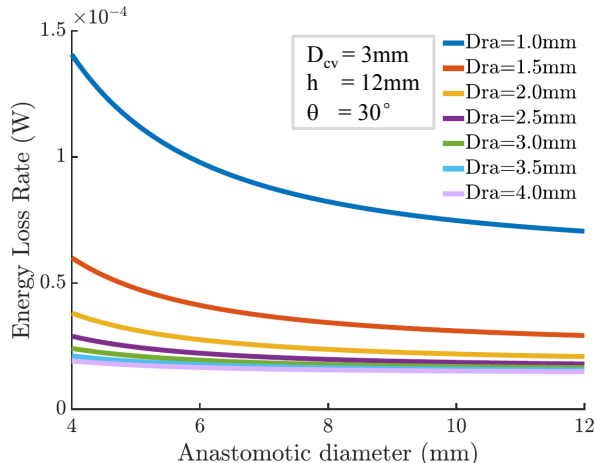

(a)

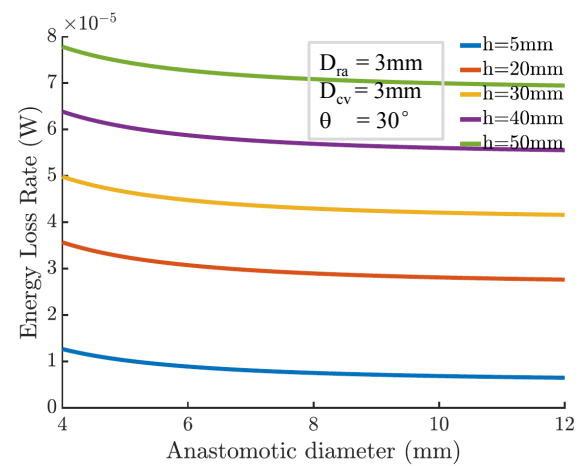

(c)

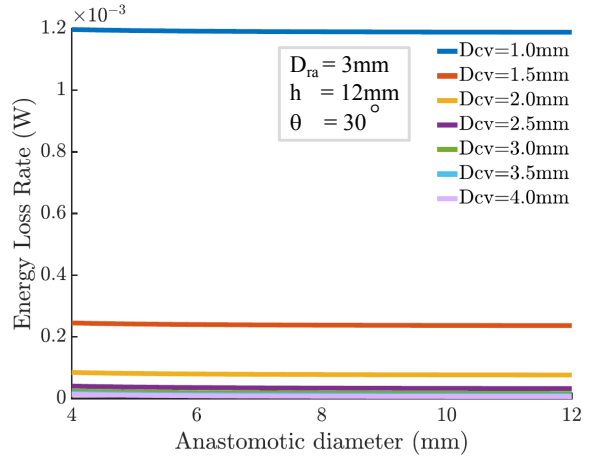

(b)

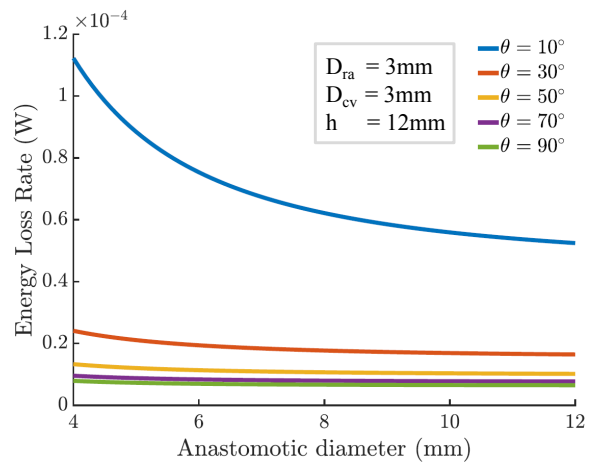

(d)

Figure 11. The relationship between energy loss $\left(E_{\text {avf }}\right)$ and anastomotic diameter $\left(D_{a}\right)$. 


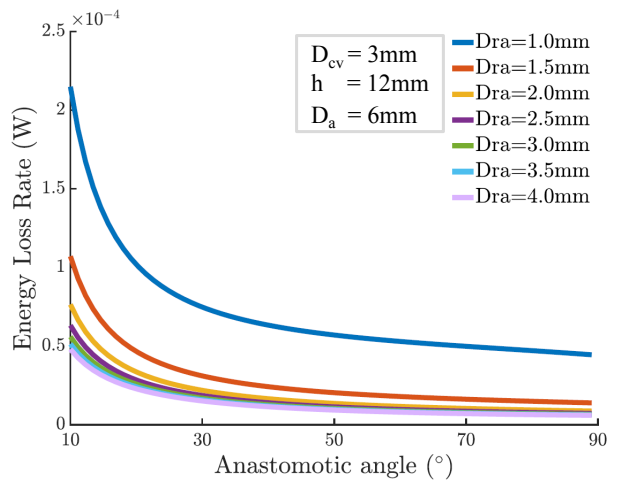

(a)

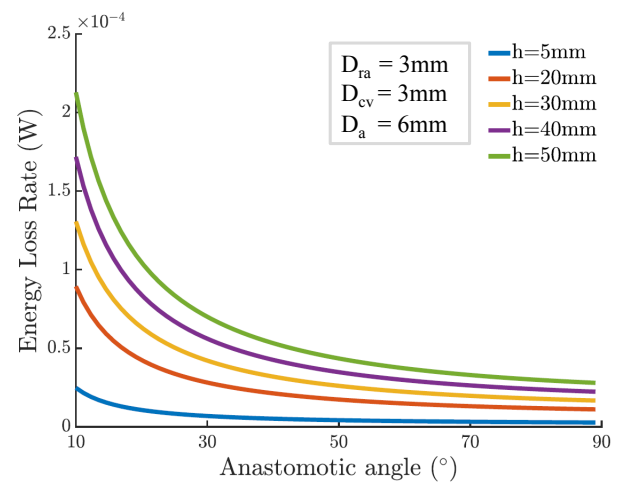

(c)

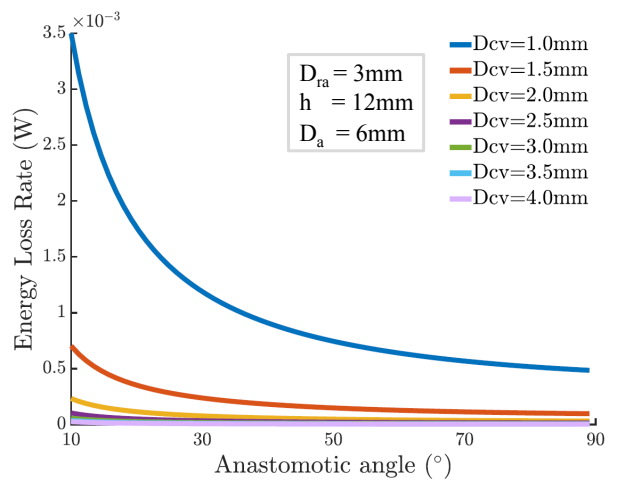

(b)

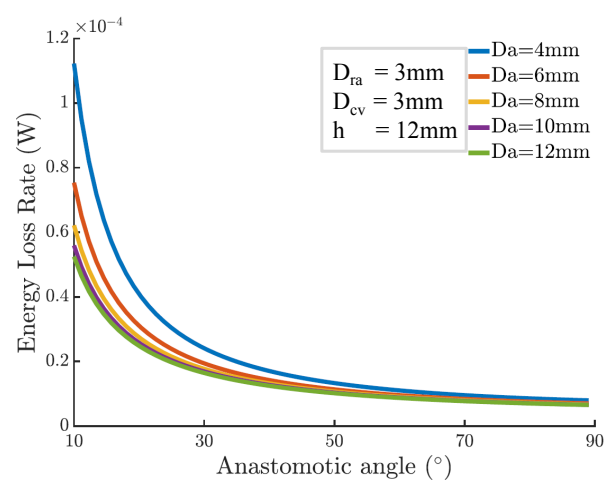

(d)

Figure 12. The relationship between energy loss $\left(E_{\text {avf }}\right)$ and anastomotic angle $(\theta)$.

2) Compared to $D_{r a}, D_{c v}$ had a more significant impact on $E_{a v e}$ Large-diameter CV $\left(D_{c v}\right)$ could effectively reduce the energy loss rate in the fistula and increase the maturity rate of RCAVF than large-diameter RA $\left(D_{r a}\right)$.

Figure 8 and Figure 9 show that when $D_{r a}$ changed, the range of variation of $E_{\text {avf }}$ was much smaller than the change caused by changing $D_{c r}$. We could also see that the curves in Figure 9 were steeper than Figure 8. Therefore, we could prove that the maturity rate of RCAVF had a significant correlation with $D_{c n}$ and the correlation with $D_{r a}$ was relatively weak. This result agreed with [19], which summarized a clinical survey carried out on 96 patients who used RCAVF for dialysis. The author concluded that fistula maturation correlated with $D_{c r}$ but there was no correlation with $D_{r a}$.

3) To increase the maturity rate of RCAVF, the choice of $\theta$ was more important than $D_{a}$.

By comparing Figure 11 with Figure 12, we could observe that variation of $E_{\text {avf }}$ with $\theta$ was more significant than $D_{a}$. When $\theta$ increased, the energy loss rate could be effectively reduced in RCAVF. It could be seen from Figure 9(c) and Figure 11(b) that when $D_{c v}$ changed, the effect of $D_{a}$ on $E_{a v f}$ was almost negligible. It could be seen from Figure 11(d) and Figure 12(d) that only when $\theta$ was very small, the $D_{a}$ had some effect on the energy loss. This phenomenon could be explained. The energy loss at the RCAVF anastomosis is mainly caused by the friction $E_{f}$ of the venous bending segment. The loss due to friction is called the major loss because it is much larger than the minor loss $\left(E_{a}\right.$ and $\left.E_{b}\right)$ due to local shape changes. The change of $\theta$ will cause the length of the venous bending segment to change and thus change the major loss, while the change of $D_{a}$ will only cause a slight change in $E_{a}$. Therefore, compared with $\theta$, the influence of $D_{a}$ on $E_{\text {avf }}$ is almost negligible.

4) When $h$ was large, it was recommended to choose a larger $D_{c v}$ or decrease the distance between the vessels to reduce $E_{\text {avf }}$

It could be observed from Figure 10(b) and Figure 10(d) that the greater the $h$, the greater the influence of $D_{c v}$ on $E_{a v f}$ Therefore, for some patients with RA and CV on different sides of the arm, the selec- 
tion of $D_{c r}$ was particularly important. If the patient's CV $\left(D_{c r}\right)$ was too small or the anastomosis design could not be achieved due to the limitation of the muscles or other tissues in the arm, in order to guarantee the maturity at this point, we could decrease the $\mathrm{h}$ (for example, releasing part of the CV in the forearm) to reduce the energy loss rate or choose other types of AVF.

\subsection{Critical Energy Loss Rate (CEL)}

To ensure the maturity and primary patency of RCAVF, the Kidney disease outcomes quality initiative (KDOQI) [1] and the European Society for Vascular Surgery (ESVS) [20] pointed out in the 2019 vascular access clinical guidelines that it was recommended to use an artery and vein with a diameter greater or equal to $2 \mathrm{~mm}$. From a systematic review [3], the author summarized 12 articles (1200 patients in total) and proposed to use CV and RA with a diameter greater than or equal to $2 \mathrm{~mm}$, and not recommended to use CV and RA with a diameter less than $1.5 \mathrm{~mm}$. In [4-6], the authors also had different proposals for the recommended $D_{c v}$ and $D_{r a}$ ranges. All these suggested critical $D_{c v}$ and $D_{r a}$ values could ensure a high RCAVF maturity rate range from $1.5 \mathrm{~mm}-2 \mathrm{~mm}$. However, no research has highlighted a precise critical value of $D_{c v}$ and $D_{r a}$ that can guarantee the maturity of RCAVF. Our analysis found that the reason for this phenomenon was that they ignored the impacts of $\theta$. It could be observed from Figure 12(a) that when $D_{c v}=3 \mathrm{~mm}, D_{a}=6 \mathrm{~mm}$, and $h=12 \mathrm{~mm}$, the $E_{a v f}$ generated when $D_{r a}=1 \mathrm{~mm}$ and $\theta=70^{\circ}$ is less than and the case when $D_{r a}=2 \mathrm{~mm}$ and $\theta=10^{\circ} \mathrm{mm}$. Consequently, we could explain why there were still mature cases when $D_{c v}$ or $D_{r a}$ was under $1.5 \mathrm{~mm}$. Therefore, to ensure the RCAVF maturity, the choice of $\theta$ was also essential while considering the vessel diameters. We proposed a Critical Energy Loss rate (CEL) concept to help surgeons choose the range of $\theta$ reasonably while considering patient's specific $D_{c r}$ and $D_{r a}$

\subsubsection{CEL Definition}

The vascular resistance generated at the anastomosis makes it difficult to increase the blood flow in RA, which will lead the blood flow in CV difficult to reach the dialysis standard, thus making the non-mature fistula. Critical energy loss rate referred to the maximum allowable energy loss rate in the RCAVF. When the energy loss rate in RCAVF was less than this threshold, the maturity of the fistula could be guaranteed.

To define the CEL, $h$ was set according to the distribution of blood vessels on the forearm of each patient. From the result (3.2.c), since $D_{a}$ had a relatively lesser impact on energy loss compared to $\theta$, we fixed it to a constant value: $6.41 \mathrm{~mm}$ [6]. To ensure the maturity of RCAVF to the greatest extent, CEL was defined as the smallest $E_{a v f}$ obtained for the critical $D_{r a}$ and $D_{c v}$ and $\theta=90^{\circ}$.

This value of CEL was calculated for the critical values of $D_{r a}$ and $D_{c v}$ as reported by [3-6]. Thus, for this analysis, $D_{r a}$ and $D_{c v}$ evolved in the range of $1.5 \mathrm{~mm}$ to $2 \mathrm{~mm}$. We were able to deduce an interval $\left[C E L_{a}, C E L_{b}\right]$ that could be used to find the best geometry guaranteeing the maturity of the fistula. These conditions were summarized in Table 4.

\subsubsection{CEL to Help for RCAVF Anastomoses Design}

In reality, $D_{r a}, D_{c n}$ and $h$ are fixed values for each patient. The purpose of the definition of CEL is to help surgeons assess, from a mechanical point of view, the usability of patients' blood vessels. In the case of usable blood vessels, the concept of critical energy loss could help design the best shape anastomosis.

Table 4. Definition of CEL.

\begin{tabular}{cccccc}
\hline & \multicolumn{5}{c}{ GPs } \\
\cline { 2 - 5 } & $D_{r a}(\mathrm{~mm})$ & $D_{c v}(\mathrm{~mm})$ & $h(\mathrm{~mm})$ & $D_{a}(\mathrm{~mm})$ & $\theta\left(^{\circ}\right)$ \\
\hline$C E L_{a}$ & 2 & 2 & Clinic data of the patient & 6.41 & 90 \\
$C E L_{b}$ & 1.5 & 1.5 & & & \\
\hline
\end{tabular}


In this analysis, we maintained $D_{a}$ at a constant value of $10 \mathrm{~mm}$, which corresponded to the value practiced by our hospital partners. $D_{r a}, D_{c n}$ and $h$ values for each patient could be measured by duplex ultrasound. Therefore, we could get the relation between $E_{\text {avf }}$ and $\theta\left(E_{a v}(\theta)\right)$, as shown in Figure 13. The black curves in Figure 13 represent the evolution with $\theta$ of the energy loss rate in RCAVF calculated using the reel $D_{r a}, D_{c r}$ and $h$ of each patient. The red and blue lines present $C E L_{a}$ and $C E L_{b}$, respectively. With $C E L_{a}$ and $C E L_{b}$, we could calculate $\theta_{a}$ and $\theta_{b}$ through the $E_{a v}(\theta)$ curve, which provided a range of critical $\theta$.

We divided the anastomosis design into three possible levels, as shown in Table 5 . When $\theta$ was greater than or equal to $\theta_{a}, E_{\text {avf }}$ was less than or equal to $C E L_{a}$ (the red area in Figure 13), this $\theta$ range was the most recommended range, and we defined it as the A-level anastomosis design. When $\theta$ was between $\theta_{a}$ and $\theta_{b}, E_{a v f}$ was between $C E L_{a}$ and $C E L_{b}$ (the blue area in Figure 13). At this time, $\theta$ was classified as $\mathrm{B}-$ level. If surgeons choose $\theta$ within this range, the performance of the patient's blood vessel wall properties should be evaluated. For patients with low vascular wall elasticity and curved blood vessels, surgeons must combine their own clinical experiences to use $\theta$ within this range before surgery. For an anastomosis design with $\theta$ smaller than $\theta_{b}, E_{a v f}$ was greater than $C E L_{b}$ (the grey area in Figure 13). This type of anastomosis design was not recommended. There was a high chance that it will cause the failure of RCAV maturation.

Not all the blood vessels could get $\theta_{a}$ and $\theta_{b}$. Through the $E_{a v f}(\theta)$ curve in Figure 13, the blood vessels used for RCAVF was divided into three types:

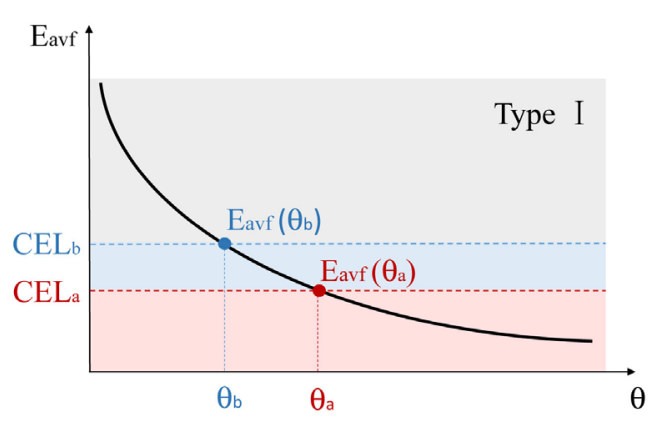

(a)

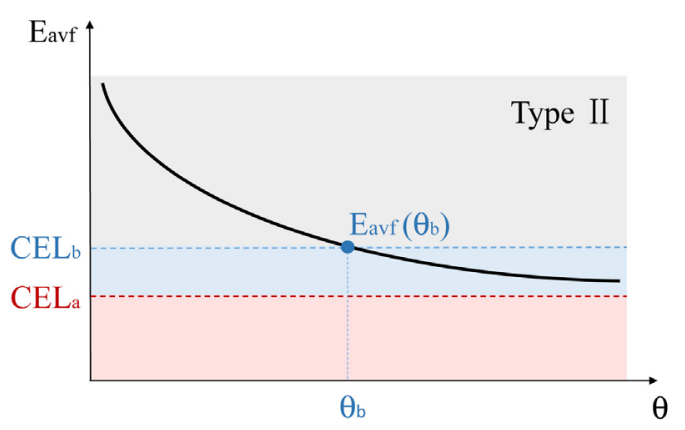

(b)

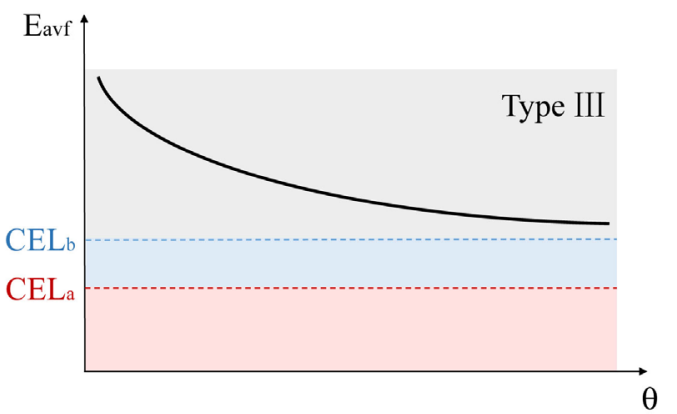

(c)

Figure 13. The relationship between $E_{\text {avf }}$ and $\theta$ regarding the critical energy loss.

Table 5. Recommended level for anastomosis design.

\begin{tabular}{ccc}
\hline Recommande Levels & Definition & Comments \\
\hline $\mathrm{C}$ & $C E L_{b} \leq E_{\text {avf }}$ & Not recommend \\
$\mathrm{B}$ & $C E L_{a}<E_{\text {avf }}<C E L_{b}$ & Must combine surgeons' clinical experiences \\
$\mathrm{A}$ & $E_{\text {avf }} \leq C E L_{a}$ & Recommend \\
\hline
\end{tabular}


Type I: $\theta_{a}$ and $\theta_{b}$ could be calculated,

Type II: Only $\theta_{b}$ could be calculated,

Type III: Neither of $\theta_{a}$ or $\theta_{b}$ could be calculated.

We recommended that surgeons choose the blood vessel Type I with an A-level anastomosis design to ensure RCVAF maturity and primary patency. For the blood vessel of Type I and Type II with B-level anastomosis design, the surgeons needed to evaluate the patient's vascular performance in advance. For RCAVF with C-level anastomosis, all three types of blood vessels were not recommended.

\section{CONCLUSIONS}

In most cases, native RCAVF is the preferred VA strongly recommended by clinical practice guidelines $[1,20]$. It has the advantages of longer survival patency, fewer complications, and lower risk of infection, making it have lower morbidity and mortality and could reduce the patient's financial burden. Nevertheless, its high early failure rate (46\%) [21] is still a big problem now. A recent study [22] proposed that the traditional vein-to-artery configuration could be changed to the artery-to-vein, thereby increasing the maturity rate and primary patency of the native fistula. Our study also concentrated on fistula configuration optimization. It was the first study that proposed to help surgeons design the fistula shapes from the perspective of energy loss, thereby increasing the RCAVF maturity rate.

Through this study, we analyzed the relationships between the energy loss rate in RCAVF and its five associated GPs, and then verified their accuracies with numerical results. It was found that $D_{a}$ and $D_{r a}$ had much less influence on energy loss than $\theta$ and $D_{c r}$, and the distance between blood vessels $h$ also had a non-negligible influence on $E_{a v 6}$ For patients who use RCAVF for dialysis, we suggested surgeons choose the vessels with the larger diameters and a small distance while increasing the angle and diameter of the anastomosis to decrease the energy loss.

To help surgeons create a RCAVF plan for patients, we defined two energy loss rate thresholds: $C E L_{a}$ and $C E L_{b}$, and used them to classify blood vessels into three types and anastomotic design into three levels. These could help surgeons access the operability of the patient's blood vessel and select the most appropriate anastomosis angle range for the patient.

This new complete model studied the different GPs. The anastomotic angle seemed to be an important parameter, more than the anastomotic diameter. This result may raise some questions about surgical theories regarding the treatment of AVF complications, such as high flow correction. Depending on the model, reducing $D_{a}$ to reduce high-flow AVF may not have real meaning. To verify this result, other studies must be conducted on mature AVF using this model.

This model can also be used to design other native AVFs and AVG (arteriovenous graft) shapes. In use, the inlet flow rate and GPs need to be reset, and the minor loss coefficients $K_{t}$ and $K_{h}$ in this model need to be changed according to relevant references when blood flow direction at the anastomosis changes.

\section{ACKNOWLEDGEMENTS}

Thanks to the Chinese government for funding this research and to the Hospices Civils de Lyon to provide the clinical data for this study and participate in this work.

\section{CONFLICTS OF INTEREST}

The authors declare no conflicts of interest regarding the publication of this paper.

\section{REFERENCES}

1. Lok, C.E., Huber, T.S., Lee, T., Shenoy, S., Yevzlin, A.S., Abreo, K., Foundation, N.K., et al. (2020) KDOQI

Clinical Practice Guideline for Vascular Access: 2019 Update. American Journal of Kidney Diseases, 75, S1-S164.

https://www.sciencedirect.com/science/article/pii/S0272638619311370 
https://doi.org/10.1053/j.ajkd.2019.12.001

2. Fung, Y.C. (2013) Biomechanics: Circulation. Springer Science \& Business Media, Berlin.

3. Kordzadeh, A., Chung, J. and Panayiotopoulos, Y.P. (2015) Cephalic Vein and Radial Artery Diameter in Formation of Radiocephalic Arteriovenous Fistula: A Systematic Review. The Journal of Vascular Access, 16, 506-511. https://doi.org/10.5301/jva.5000413

4. Parmar, J., Aslam, M. and Standfield, N. (2007) Pre-Operative Radial Arterial Diameter Predicts Early Failure of Arteriovenous Fistula (AVF) for Haemodialysis. European Journal of Vascular and Endovascular Surgery, 33, 113-115. https://www.sciencedirect.com/science/article/pii/S1078588406004618 https://doi.org/10.1016/j.ejvs.2006.09.001

5. Bashar, K., Clarke-Moloney, M., Burke, P.E., Kavanagh, E.G. and Walsh, S.R. (2015) The Role of Venous Diameter in Predicting Arteriovenous Fistula Maturation: When Not to Expect an AVF to Mature According to Pre-Operative Vein Diameter Measurements? A Best Evidence Topic. International Journal of Surgery, 15, 95-99. https://www.sciencedirect.com/science/article/pii/S1743919115000540

https://doi.org/10.1016/j.ijsu.2015.01.035

6. Gjorgjievski, N., Dzekova-Vidimliski, P., Trajcheska, L., Stojanoska, A., Selim, G., Rambabova-Bushljetik, I., Ivanovski, N., et al. (2020) Impact of Preoperative Arterial and Venous Diameter on Achievement of the Adequate Blood Flow in Arteriovenous Fistula for Hemodialysis. Therapeutic Apheresis and Dialysis: Official Peer-Reviewed Journal of the International Society for Apheresis, the Japanese Society for Apheresis, the Japanese Society for Dialysis Therapy, 25, 273-281. https://europepmc.org/article/med/32749076 https://doi.org/10.1111/1744-9987.13570

7. Jackson, Z.S., Ishibashi, H., Gotlieb, A.I. and Langille, B.L. (2001) Effects of Anastomotic Angle on Vascular Tissue Responses at End-to-Side Arterial Grafts. Journal of Vascular Surgery, 34, 300-307.

https://www.sciencedirect.com/science/article/pii/S0741521401626168 https://doi.org/10.1067/mva.2001.115815

8. Pousset, Y., Lermusiaux, P., Berton, G., Le Gouez, J.M. and Leroy, R. (2006) Numerical Model Study of Flow Dynamics through an End-to-Side Anastomosis: Choice of Anastomosis Angle and Prosthesis Diameter. Annals of Vascular Surgery, 20, 773-779. https://link.springer.com/article/10.1007\%2Fs10016-006-9125-9 https://doi.org/10.1007/S10016-006-9125-9

9. Freshwater, I.J., Morsi, Y.S. and Lai, T. (2006) The Effect of Angle on Wall Shear Stresses in a LIMA to LAD Anastomosis: Numerical Modelling of Pulsatile Flow. Proceedings of the Institution of Mechanical Engineers, Part H: Journal of Engineering in Medicine, 220, 743-757. https://doi.org/10.1243/09544119JEIM126

10. Ene-Iordache, B., Cattaneo, L., Dubini, G. and Remuzzi, A. (2013) Effect of Anastomosis Angle on the Localization of Disturbed Flow in "Side-to-End" Fistulae for Haemodialysis Access. Nephrology Dialysis Transplantation, 28, 997-1005. https://academic.oup.com/ndt/article/28/4/997/1852964?login=true https://doi.org/10.1093/ndt/gfs298

11. Prouse, G., Stella, S., Vergara, C., Engelberger, S., Trunfio, R., Canevascini, R., Giovannacci, L., et al. (2019) Computational Analysis of Turbulent Haemodynamics in Radiocephalic Arteriovenous Fistulas with Different Anastomotic Angles. https://www.mate.polimi.it/biblioteca/add/qmox/08-2019.pdf https://doi.org/10.1016/j.avsg.2020.04.004

12. Jůza, T. and Vlk, D. (2018) Immediate Effect of Physical Exercise on Blood Flow Velocity in Radial Artery in Young Adults. Lékař a Technika-Clinician and Technology, 48, 118-122. https://ojs.cvut.cz/ojs/index.php/CTJ/article/view/5117

13. Wilson, K.E., Tat, J. and Keir, P.J. (2017) Effects of Wrist Posture and Fingertip Force on Median Nerve Blood Flow Velocity. BioMed Research International, 2017, Article ID: 7156489. 
https://www.hindawi.com/journals/bmri/2017/7156489

https://doi.org/10.1155/2017/7156489

14. Beniwal, S., Bhargava, K. and Kausik, S.K. (2014) Size of Distal Radial and Distal Ulnar Arteries in Adults of Southern Rajasthan and Their Implications for Percutaneous Coronary Interventions. Indian Heart Journal, 66, 506-509. https://www.sciencedirect.com/science/article/pii/S0019483214002636 https://doi.org/10.1016/j.ihj.2014.08.010

15. Idel'Cik, I.E. (1968) Handbook of Hydraulic Resistance. Washington DC.

16. Bassett, M.D., Winterbone, D.E. and Pearson, R.J. (2001) Calculation of Steady Flow Pressure Loss Coefficients for Pipe Junctions. Proceedings of the Institution of Mechanical Engineers, Part C: Journal of Mechanical Engineering Science, 215, 861-881. https://doi.org/10.1177/095440620121500801

17. Ochsner Jr., A.L.T.O.N., Colp Jr., R.A.L.P.H. and Burch, G.E. (1951) Normal Blood Pressure in the Superficial Venous System of Man at Rest in the Supine Position. Circulation, 3, 674-680. https://doi.org/10.1161/01.CIR.3.5.674

18. Moukalled, F., Mangani, L. and Darwish, M. (2016) The Finite Volume Method in Computational Fluid Dynamics. Vol. 113, Springer, Berlin, 10-1007. https://doi.org/10.1007/978-3-319-16874-6

19. Khavanin Zadeh, M., Gholipour, F., Naderpour, Z. and Porfakharan, M. (2012) Relationship between Vessel Diameter and Time to Maturation of Arteriovenous Fistula for Hemodialysis Access. International Journal of Nephrology, 2012, Article ID: 942950. https://www.hindawi.com/journals/ijn/2012/942950 https://doi.org/10.1155/2012/942950

20. Schmidli, J., Widmer, M.K., Basile, C., de Donato, G., Gallieni, M., Gibbons, C.P. and ESVS Guidelines Committee (2018) Editor's Choice-Vascular Access: 2018 Clinical Practice Guidelines of the European Society for Vascular Surgery (ESVS). European Journal of Vascular and Endovascular Surgery, 55, 757-818. https://www.ejves.com/article/S1078-5884(18)30080-7/abstract https://doi.org/10.1016/j.ejvs.2018.02.001

21. Allon, M., Lockhart, M.E., Lilly, R.Z., Gallichio, M.H., Young, C.J., Barker, J., Robbin, M., et al. (2001) Effect of Preoperative Sonographic Mapping on Vascular Access Outcomes in Hemodialysis Patients. Kidney International, 60, 2013-2020. https://www.sciencedirect.com/science/article/pii/S0085253815480846 https://doi.org/10.1046/j.1523-1755.2001.00031.x

22. Bai, H., Sadaghianloo, N., Gorecka, J., Liu, S., Ono, S., Ramachandra, A.B., Dardik, A., et al. (2020) Artery to Vein Configuration of Arteriovenous Fistula Improves Hemodynamics to Increase Maturation and Patency. Science Translational Medicine, 12, eaax7613. https://stm.sciencemag.org/content/12/557/eaax7613.abstract https://doi.org/10.1126/scitranslmed.aax7613 